\section{How the world is (not) handling surplus doses and expiring vaccines}

In this feature article published on 25 August 2021 (doi:) the original version of the figure included summary EU data as well as individual countries. This has been removed.

Also, data on the number of doses expiring before the end of 2021 had been combined with those expiring by the end of 2022. The new version presents only doses expiring by the end of 2022, thus avoiding double counting of the doses expiring before the end of 2021.

We have added this caption to the graphic contained in the main article: "This diagram shows the number of surplus doses that could accumulate by the end of 2022 in the 10 locations with the greatest potential waste."

In the PDF attached as a separate infographic, we have replaced this sentence: "The diagram below shows the number of surplus doses due to expire by the end of 2022 in the 10 locations with the worst potential problems." The new version reads: "The diagram below shows the number of surplus doses that could accumulate by the end of 2022 in the 10 locations with the greatest potential waste." 